\title{
Cooperação interprofissional e a Reforma Sanitária no Brasil: implicações para o modelo de atenção à saúde
}

\author{
Interprofessional collaboration and the Brazilian Sanitary \\ Reform: implications for delivery of healthcare
}

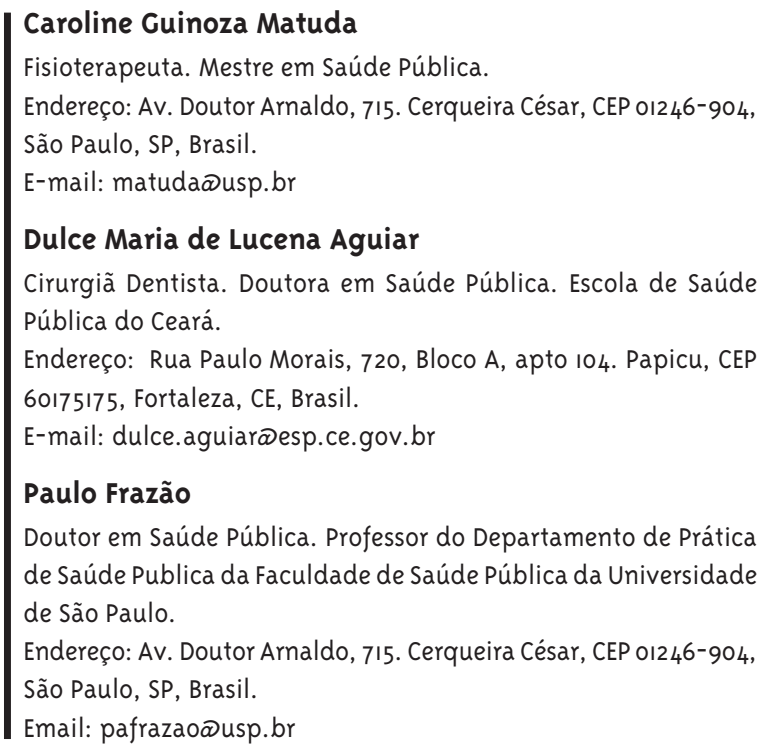

\section{Resumo}

As transformações sanitárias, sociais e econômicas ocorridas no Brasil recolocaram antigos problemas e introduziram outros novos para a área de recursos humanos em saúde. Entre os desafios inéditos, destacam-se aqueles decorrentes da implementação de estratégias de reorientação do modelo de atenção à saúde que exigem intensificação dos processos de interação profissional. 0 objetivo deste artigo foi sistematizar os conhecimentos relativos à cooperação interprofissional como um objeto de pesquisa, destacando sua relevância para a compreensão das relações entre os trabalhadores na produção do cuidado no contexto da reforma do sistema de saúde brasileiro. Aspectos históricos que marcaram as reformas do setor saúde no mundo, e no Brasil em particular, foram apresentados. Tomando-se por base os conhecimentos produzidos por estudos de revisão sobre o assunto, foram sistematizados aspectos que conformam a teoria sobre a cooperação interprofissional com foco nas definições, nos modelos teóricos e nos resultados observados. Para superar as dificuldades impostas pela necessidade de tornar a atenção à saúde mais efetiva, eficiente e equitativa, é indicada uma abordagem dirigida à compreensão dos processos e das relações imbricadas no problema que considere os profissionais como agentes atuantes, dotados de uma prática interessada, admitindo-se que tanto os esquemas de percepção como as organizações e a estrutura possuem gênese social. A compreensão das forças que orientam o conflito e a cooperação interprofissional pode subsidiar a formulação de estratégias para melhorar a produção do cuidado no âmbito do sistema de saúde no Brasil.

Palavras-chave: Relações interprofissionais; Comportamento cooperativo; Atenção primária à saúde. 


\section{Abstract}

The social, economic and sanitary changes in Brazil reinserted earlier problems and brought news ones for the health human resources area. Among the new challenges, it is important to underline those implicated to reorientation strategies of the health care model which has required more intensive process of professional interaction. The aim of this article was to review the knowledge related to interprofessional collaboration as a research issue highlighting its importance for understanding of the relations among workers in the production of care within Brazilian health system. Historical characteristics that marked the health sector reforms in the world and particularly in the Brazil were summed up. Based on review studies, knowledge about interprofessional collaboration were summarized focusing definitions, theoretical models and the observed results. An approach directed to comprehension of process and relations implicated to the problem is indicated in order to surmount the barriers connected to the need for a more effective, efficient and equitable health care model. For this it is necessary to admit the professional as a social agent bearer of an interested practice considering that thinking frameworks as organizations and overall structure denote social construct. The understanding of the forces that guide the interprofessional conflict and collaboration may subsidize the formulation of strategies for improving healthcare in Brazilian health system.

Keywords: Interprofessional Relations; Cooperative Behavior; Primary Health Care.

\section{Introdução}

A importância dos sistemas de saúde enquanto um mecanismo social para assegurar níveis elevados de bem-estar coletivo foi um dos principais legados da Conferência Internacional de Cuidados Primários em Saúde, realizada em 1978 pela Organização Mundial da Saúde (OMS) (Cueto, 2004).

A saúde como um direito humano fundamental e a responsabilidade dos Estados nacionais, das sociedades e das pessoas para alcançar essa relevante meta social foram reafirmadas pelos delegados e representantes de países e entidades presentes, que, entre outros aspectos, destacaram a importância da relação entre saúde e desenvolvimento, enfatizando a necessidade de estabelecer a atenção primária como parte de um sistema nacional de saúde, integrado em seus vários níveis de atenção e em coordenação com outros setores (Rivero, 2003).

Nos anos 1980, as instituições e os serviços de saúde foram atravessados por várias medidas de reforma do setor, entre as quais a descentralização das ações e serviços. Embora a força de trabalho dos serviços de saúde seja responsável pela maior proporção dos gastos em saúde e represente um aspecto essencial para assegurar a qualidade das ações, observa-se desse período até o início do século XXI que as reformas do setor deixaram um legado de subinvestimento crônico em relação aos recursos humanos. Nessas duas décadas de reforma econômica e setorial, assistiu-se, de modo geral, ao corte de despesas, ao congelamento de salários e recrutamento de pessoal, incluindo sucateamento de equipamentos e suprimentos nos ambientes de trabalho (Narasimhan e col., 2004).

No caso do Brasil, as transformações decorrentes do processo de descentralização das ações e serviços de saúde e aquelas observadas a partir da implementação de medidas relacionadas a uma agenda de reforma do Estado, nos anos 1990, recolocaram antigos problemas e introduziram outros novos para a área de recursos humanos em saúde (Pierantoni, 2001). Entre os desafios inéditos, destacam-se aqueles decorrentes de estratégias de reorientação do modelo de atenção. Um dos esforços nessa direção diz respeito à implantação da Estratégia Saúde da Família (ESF) e dos Núcleos de Apoio à Saúde da Família (NASF), na primeira década do século do XXI, 
que expressam um conjunto de recursos dirigido à reorientação do modelo de atenção, representando importante expansão dos postos de trabalho para as categorias profissionais presentes no sistema de saúde brasileiro e crescente desafio para a área de recursos humanos (Brasil, 2009).

A reorientação do modelo de atenção, no contexto brasileiro, implica várias mudanças que têm por propósito superar o modelo biomédico, com foco na assistência à doença, em seus aspectos individuais e biológicos, centrado no procedimento, nas especialidades médicas e no hospital. Por meio da reorganização das práticas de saúde, sob uma abordagem interdisciplinar com vista à integralidade da atenção, pretende-se a mudança para um modelo centrado no território e nas necessidades de saúde do paciente (Morosini e Corbo, 2007). Nessa nova configuração, as limitações que caracterizam o conhecimento biomédico e a prática autônoma seriam reconhecidas. Para abordar as necessidades do paciente, haveria, entre outras exigências, de se considerar a contribuição de outros profissionais da área da saúde, do serviço social e de outros sistemas de ação social para a produção do cuidado em saúde (D’Amour e col., 2005). Entretanto, é pequeno o número de estudos sobre esse tema, no âmbito do sistema de saúde brasileiro.

Alguns especialistas chamam atenção para as exigências de articulação do conhecimento nas equipes de saúde envolvendo todas as categorias profissionais. Destacam a necessidade do aprendizado para lidar com as dinâmicas relacionais e os processos de interação com o outro, abrangendo não apenas a relação entre as equipes e os usuários, mas também a relação entre os membros das equipes (Franco e Merhy, 2007). Nesse âmbito, as formas de comunicação e de interação entre os profissionais podem ser importante fonte de conflitos ou de cooperação interprofissional.

O objetivo deste artigo foi sistematizar os conhecimentos relativos à interação do pessoal de saúde, com destaque para os estudos de revisão que abordam a cooperação interprofissional como um objeto de pesquisa, destacando sua relevância para a compreensão das relações entre os trabalhadores na produção do cuidado no contexto da reforma do sistema de saúde brasileiro.

\section{Procedimentos Metodológicos}

Para fundamentar a análise e o contexto geral em que está inserida essa problemática, recorreu-se a um breve histórico dos antecedentes que marcaram as reformas do setor saúde no mundo e no Brasil, em particular. Em seguida, são apresentados aspectos que conformam a teoria sobre cooperação interprofissional, tomando-se por base os conhecimentos produzidos por estudos de revisão sobre o assunto. Para seleção dos estudos de revisão foram consultadas as seguintes bases de dados: Embase; PubMed/Medline, Lilacs e Centro de Revisão e Disseminação da Universidade de York, esta última uma importante fonte de revisões, mantida pelo Instituto Nacional para Pesquisa em Saúde do Reino Unido, para subsidiar tomadores de decisão. A busca foi efetuada empregando-se a expressão "cooperação interprofissional" (interprofessional collaboration) no campo título, no período de 2000 a 2010. Todos os trabalhos foram lidos na íntegra, sendo incluídos apenas os estudos de revisão que abordavam os seguintes temas: conceito e classificação, determinantes, efeitos, modelos teóricos e desafios futuros.

Os autores finalizam o artigo com uma apreciação das potencialidades que a abordagem do objeto pode oferecer para a compreensão das relações entre os trabalhadores na produção do cuidado diante das exigências de mudar o modelo de atenção à saúde.

\section{As Reformas do Setor Saúde}

A Conferência de Alma-Ata foi realizada em 1978 em um período marcado por intensas turbulências provocadas pela crise do petróleo. 0 aumento dos preços dos combustíveis e a alta do dólar e dos juros levaram a uma queda de liquidez nas reservas internacionais e a uma forte pressão sobre os Estados nacionais, redundando em importante crise fiscal e aumento do déficit dos Estados para honrar seus compromissos (Sevcenko, 2001).

Nos debates para responder à crise predominou a visão de que era necessário oferecer maior dinamismo ao sistema financeiro mundial e, com isso, houve a liberalização dos fluxos de capital. A possibilidade de multiplicar filiais, nas mais diversas partes do planeta, propiciou um enorme poder de barganha das grandes empresas e corporações. 
Os Estados nacionais demandavam investimentos e geração de postos de trabalho. As corporações, em contrapartida, exigiam a desregulamentação dos mercados (Sevcenko, 2001).

A razão para os delegados de Alma-Ata enfatizarem a importância dos sistemas de saúde estava associada ao reconhecimento de que, em geral, os sistemas universais dão ênfase à atenção básica e à prevenção, e, assim, alcançam melhores condições de saúde com menos recursos. Em sistemas segmentados, nos quais prevalece o setor privado de assistência, dá-se ênfase à atenção especializada, sendo também mais custosos e com piores níveis de saúde do que sistemas universais. As recomendações de Alma-Ata exerceram intensa influência na saúde pública mundial, gerando desdobramentos em diferentes áreas (Buss, 200o). Entretanto, ao lado da difusão da estratégia da atenção primária à saúde (APS), centros hegemônicos da economia mundial revalorizavam o mercado como mecanismo privilegiado para a alocação de recursos, questionando a responsabilidade estatal na provisão de bens e serviços de saúde (Paim e Almeida-Filho, 1998).

A desregulamentação dos mercados e o desenvolvimento de novas tecnologias microeletrônicas, entre outros aspectos, criaram condições para a separação entre as práticas financeiras e os empreendimentos econômicos. As grandes empresas ganharam maior mobilidade, possibilidade de redução da mão de obra e capacidade de negociação. Os objetivos corporativos, preconizados naquele período, poderiam ser sumarizados em: menos impostos, menos Estado e menos postos de trabalho (Sevcenko, 2001).

A chamada "crise fiscal do Estado" se agravou nos anos 1980. A consequência desse agravamento foi o corte de gastos públicos (Sevcenko, 2001) e a implementação de estratégias de reforma do setor saúde, entre as quais a descentralização das ações e serviços de saúde.

A descentralização ganhou paulatinamente adeptos, tanto no campo dos que defendiam o mercado como mecanismo de regulação da oferta e demanda de serviços de saúde, quanto no terreno dos defensores do Estado como organismo de gestão. Do ponto de vista do pensamento liberal, argumentava-se que a redução do Estado propiciaria mais eficiên- cia aos processos produtivos, sendo que no campo da saúde a privatização dos serviços permitiria sua gestão em regime de competição regulada. Do ponto de vista do pensamento social, vislumbrava-se no processo de descentralização uma rica oportunidade para aumentar o impacto do sistema, a justiça social e a democracia. Em comum, essas diferentes visões propugnavam a transferência de poder do nível central para outros espaços.

Passados 30 anos da Declaração de Alma-Ata, o Relatório Mundial de Saúde, divulgado pela OMS em 2008, reacende a importância da APS enquanto estratégia para impulsionar as reformas dos sistemas de saúde nos diferentes países. Ao mesmo tempo em que se denuncia o hospitalocentrismo (ênfase em cuidados especializados), a fragmentação e a comercialização da assistência como tendências que comprometem a resposta dos sistemas de saúde, apresenta no documento uma defesa explícita dos sistemas universalizados. Além disso, são identificados eixos em torno dos quais é possível tornar a APS mais efetiva, eficiente e equitativa, sendo reafirmada a necessidade de considerar a saúde em todas as políticas sociais e econômicas (OMS, 2008).

A abordagem interdisciplinar e a busca da integralidade nos sistemas universais trazem para a agenda a necessidade da adoção de estratégias para melhorar a interação entre os profissionais, para uma atenção centrada nas necessidades dos pacientes e das comunidades e que alcance melhores condições de saúde.

As discussões na área da saúde sobre a colaboração interprofissional começaram na primeira metade do século XX. Porém, a importância da temática nas produções científicas e nos projetos de prática multiprofissional aumentou significativamente somente após a Segunda Guerra Mundial (Baldwin, 2007). Na década de 1970, seguindo a mesma direção das discussões sobre a APS, a OMS passa a considerar a educação interdisciplinar como estratégia complementar aos programas tradicionais de ensino na formação dos profissionais de saúde (Oandasan e col., 2004).

Esse movimento influenciou diversos países, como o Reino Unido, onde a estratégia da cooperação interprofissional faz parte do planejamento e da política de governo adotada pelo seu sistema de saúde. 
Tal política governamental reforça a necessidade de modernização e mudança do sistema de formação profissional e de serviços de saúde, enfatizando a importância da cooperação e a necessidade de partilhar conhecimentos e aprendizados (Leathard, 2003). No Canadá, iniciativas na formação e no exercício profissional apoiadas por políticas governamentais e acordos entre os sistemas de saúde e educação são exemplos de cooperação interprofissional (Oandasan e col., 2004).

Em 2007, a OMS anuncia a criação de um grupo de estudos em educação interprofissional e prática colaborativa, respondendo a uma necessidade de formação de mais profissionais para a área da saúde de maneira interdisciplinar e elaboração de novas estratégias de ensino, reconhecendo a necessidade dessa temática na construção de sistemas e programas de saúde na atualidade (Yan e col., 2007). Essas estratégias são endossadas em 2010 com o lançamento da publicação Framework for action on interprofessional education \& collaborative practice (WHO, 2010), afirmando a colaboração interprofissional como um recurso que pode ser mobilizado para elevar a efetividade dos sistemas de saúde. Como estratégia inovadora, ela pode desempenhar um importante papel para enfrentar problemas do modelo de atenção e da força de trabalho em saúde, contribuir para fortalecer o sistema de saúde e melhorar os resultados obtidos.

\section{A Reforma do Setor Saúde no Brasil}

No Brasil, as discussões para a adesão às propostas da APS tiveram início na década de 1970, acompanhando o movimento internacional. Os debates confluem para a necessidade da reforma do setor e se refletem na aprovação da descentralização como uma das diretrizes do Sistema Único de Saúde (SUS), criado pela Constituição de 1988.

Formulações de especialistas brasileiros, como Mendes (1996), desdobram os elementos conceituais e conferem novos sentidos à descentralização, para além de uma visão meramente racionalizadora de uma assistência médica aos não integrados economicamente. São destacadas as dimensões políticas (espaços de disputa entre diferentes atores), ideológicas (disputa de diferentes concepções de modelo de atenção) e técnicas (disputa de diferentes arranjos tecnológicos) da estratégia em curso.

Nos anos 1990, assiste-se ao abandono da concepção de seguridade social, prevista na Constituição de 1988, que preconiza um orçamento único e com diversidade de fontes de financiamento para os seus componentes de saúde, assistência e previdência social. Ao mesmo tempo, medidas de reforma do Estado impulsionam a transferência da execução de serviços de educação, saúde, cultura e pesquisa científica a um setor denominado de público nãoestatal, sem que regras para o seu funcionamento e prestação de contas sejam claramente definidas. A dependência do país por empréstimos externos se reflete, entre outros aspectos, em um ajuste fiscal que implica forte restrição ao gasto público em saúde (Noronha e Soares, 2001).

Em 1993 é criado o Programa Saúde da Família (PSF). É por meio dele que a atenção básica começa a ser inserida na agenda de prioridades do governo brasileiro como uma exigência, entre outras, necessária à reestruturação do modelo de atenção à saúde. Em sua fase inicial, o propósito foi colaborar na organização do SUS e na municipalização da saúde em áreas de maior risco social. Os princípios e as diretrizes da APS em sua versão mais abrangente são assumidos mais tarde, quando o programa afirma-se como estratégia articulada aos princípios da reforma e tem a possibilidade de contar com a participação de outros profissionais da saúde e do serviço social, como os das equipes de saúde bucal e das equipes do núcleo de apoio à saúde da família (NASF), na perspectiva da universalidade e integralidade da atenção (Cueto, 2004; Borges e Baptista, 2010; Brasil, 2009).

Nessa proposta, o processo de trabalho deve ser conduzido por equipes de saúde compostas por diferentes profissionais, refletindo a ampliação da concepção do processo saúde-doença, por meio da oferta de tecnologias específicas de APS e da organização das ações a partir das necessidades do território (Starfield e Shi, 2002; Giovanella e Mendonça, 2008).

Os profissionais deverão reorganizar suas práticas de modo que o diagnóstico de saúde da comunidade, componente do processo de planejamento e programação local de suas ações, seja realizado a 
partir de uma abordagem interdisciplinar que leve em consideração os determinantes sociais da saúdedoença, a integralidade da atenção, a resolubilidade e a intersetorialidade, entre outros (Brasil, 1997).

Passados mais de quinze anos da implantação da Estratégia Saúde da Família (ESF), diversos autores (Mendes, 2002; Campos, 2007; Franco e Merhy, 2007) têm analisado aspectos referentes à organização e estrutura do modelo brasileiro de APS. Embora considerem que a implementação da ESF se configure como um importante desafio da gestão, apontam, sob diferentes pontos de vista, vários obstáculos relacionados à necessidade de se estabelecer um processo de compartilhamento de responsabilidades e ampliar conceitos, conhecimentos e tecnologias que ainda não são de pleno domínio pelos profissionais e gestores.

A oferta de profissionais preparados para a atuação nesse modelo de APS é baixa. A formação profissional ainda é marcada pelo modelo convencional de atenção, baseado nas especialidades médicas e em um sistema de saúde fragmentado, com pequeno grau de coordenação e planejamento da assistência (Mendes, 2002; Campos, 2007).

Apesar de existirem diretrizes para a reorganização dos serviços, ainda permanecem desafios a serem superados, pois esse arranjo altera as relações de poder entre profissionais, usuários e gestores. Uma cultura médico-centrada permanece orientando a organização do processo de trabalho das equipes, que tendem a operar sob a lógica da produção de procedimentos. Uma mudança efetiva do modelo assistencial requer o desenvolvimento de ações que reorganizem as práticas das equipes em seus processos decisórios no cotidiano de trabalho (Franco e Merhy, 2007).

Para isso, Franco e Merhy (2007) reconhecem a necessidade de articulação do conhecimento nas equipes de saúde (não apenas do médico) para o aprendizado das dinâmicas relacionais e interação com o outro (usuário ou integrante da equipe). Consideram necessário avançar no domínio do uso das tecnologias leves (principalmente as de comunicação e interação); no desenvolvimento de novos conhecimentos técnicos, novas configurações tecnológicas do trabalho em saúde, outra micropolítica para esse trabalho e nova ética que o conduza.
Do ponto de vista do financiamento federal, os profissionais que podem compor a equipe de saúde da família são: médico generalista, enfermeiro, auxiliares de enfermagem e agentes comunitários de saúde; e na equipe de saúde bucal: o cirurgiãodentista, o técnico e o auxiliar em saúde bucal. A composição das equipes de NASF é definida pelos gestores municipais, mediante demandas reconhecidas em cada território e outros critérios de prioridades. Os profissionais que podem compor a equipe de apoio são: assistente social, educador físico, farmacêutico, fisioterapeuta, fonoaudiólogo, médico acupunturista, médico ginecologista, médico homeopata, médico pediatra, médico psiquiatra, psicólogo, nutricionista e terapeuta ocupacional (Brasil, 2009).

O trabalho no NASF é organizado e desenvolvido a partir da utilização de algumas ferramentas, como o apoio matricial, dispositivo que opera como uma retaguarda especializada para equipes da ESF. É um trabalho complementar que oferece suporte assistencial e técnico-pedagógico para a construção compartilhada de projetos terapêuticos entre as equipes (Campos e Domitti, 2007). Tem por objetivo ampliar a clínica com a agregação, de forma dialógica, de outras especialidades e profissões, para uma abordagem integral. Para que o apoio matricial se efetive é necessário um elevado grau de cooperação interprofissional. Pretende, ainda, assegurar uma maior eficácia e eficiência ao trabalho em saúde, investindo também na autonomia dos usuários dos serviços (Campos e Domitti, 2007). Por reforçar a necessidade da articulação do conhecimento nas equipes de saúde, do aprimoramento da interação entre os integrantes das equipes, do desenvolvimento de novos conhecimentos e da prática de novos processos de trabalho, o NASF se constitui como um recurso para a mudança do modelo de prática, em um contexto ainda marcado pelo modelo biomédico de atenção.

A coletivização e aumento da variedade de profissionais nas equipes de saúde, observados neste contexto, são algumas das principais características dos processos de trabalho em saúde na atualidade, cuja finalidade é assegurar a integralidade da atenção. Uma das exigências para oferecer respostas adequadas às necessidades de saúde da população 
é o trabalho associado interdependente, em regime de cooperação, seja em uma unidade isolada (equipe) ou em uma organização, instituição ou sistema (Leathard, 2003).

Maior variedade de profissionais na equipe de saúde modifica a estrutura do trabalho, mas não garante, por si só, a mudança requerida no processo de trabalho e a qualificação da assistência (Shaw e col., 2005). Para tanto, é preciso avançar na compreensão do processo nas equipes de saúde, olhando para o trabalho e a maneira como os profissionais cooperam entre si (Silva e Trad, 2005; Franco e Merhy, 2007), a fim de subsidiar a gestão dos serviços de saúde.

\section{Cooperação Interprofissional}

Foram identificados nas bases consultadas 11 estudos de revisão que apresentavam o termo "cooperação interprofissional" no campo título, entre os anos 2000 e 2010. Desses, seis foram incluídos (D`Amour e col., 2005; San Martin-Rodriguez e col., 2005; Zwarenstein e col., 2009; Martin e col., 2010; McDonald e McCallin, 2010; Mitchell e col., 2010). Cinco foram descartados porque abordavam aspectos relacionados à educação interprofissional (Goodman, 2004), às oportunidades de estágio para estudantes (Smith e Seeley, 2010), à cooperação voltada para pacientes terminais (Reese e Sontag, 2001) e a uma área profissional específica (Kenny, 2002). O último não foi incluído porque dizia respeito à relação entre colaboração interprofissional e tradução do conhecimento, uma categoria de investigação que envolve troca ativa de informação entre pesquisadores e usuários, a fim de acelerar a apropriação dos benefícios pela sociedade (Zwarenstein e Reeves, 2006).

\section{Conceito e Classificação}

A cooperação interprofissional pode ser definida como o conjunto de relações e interações que acontecem entre profissionais que trabalham juntos, no âmbito de equipes de saúde. É um processo complexo, de múltiplos determinantes, voluntário e dinâmico, que implica constante negociação (D’Amour e col., 2005).

Em revisão de literatura, D'Amour e colaboradores (2005) identificaram termos relativos à definição do conceito de cooperação interprofissional, classificados de acordo com as seguintes palavras-chave:
(1) partilha (sharing), descrita como divisões de responsabilidades, compartilhamento de tomada de decisão; (2) parceria (partnership), referindo-se a relações de afinidade, comunicação aberta, respeito e confiança mútuos; (3) interdependência (interdependency), mútua dependência, participação de cada profissional nos projetos terapêuticos; e (4) poder (power), empoderamento de cada integrante, que tem seu poder/valor reconhecido pela equipe. Para esses autores, o agrupamento de profissionais em equipes de saúde não leva necessariamente à colaboração. Para que esta ocorra, é necessário o desenvolvimento de processos colaborativos no âmbito das equipes, trabalhando as dinâmicas relacionais, bem como a relação com os demais determinantes.

Para alguns especialistas, a prática cooperada relaciona-se com um compartilhamento de poder entre os parceiros de trabalho, uma busca de objetivos pactuados em discussões e negociações, uma participação ativa e um envolvimento do profissional nos processos de trabalho coletivo (Feeley e col., 2005). Algumas características, como dinamismo profissional, capacidade de compartilhar poder, resolutividade, flexibilidade, capacidade de respeitar mutuamente e disposição para aprender, podem facilitar a prática cooperada (Oandasan e col., 2004). A colaboração é mais propensa a acontecer quando os profissionais partilham interesses, valores e filosofias similares (Gaboury e col., 2009).

\section{Determinantes}

Em revisão sobre os determinantes da cooperação foram identificadas três categorias de análise (San Martin-Rodriguez e col., 2005). A primeira refere-se aos fatores sistêmicos, relativos a elementos externos às organizações (sistemas social, cultural, educacional e profissional). Nessa categoria ressalta-se a importância da distribuição igualitária de poder entre os membros das equipes e a existência de uma cultura dominante da autonomia profissional, que tende a aumentar o individualismo e especialização em detrimento da cooperação. A segunda refere-se aos fatores organizacionais, como o tipo de estrutura organizacional (centralizada ou descentralizada; vertical ou horizontal), a filosofia da organização, o apoio administrativo e os recursos para o trabalho que podem potencializar ou impedir a cooperação, dependendo de seus termos. A terceira categoria 
refere-se aos fatores interacionais, como a receptividade aos ideais da cooperação e o compromisso com a prática cooperada; a confiança pessoal e na equipe/ colega; a comunicação efetiva e o respeito mútuo.

Os determinantes apontados no estudo não se mostram suficientes se analisados de forma isolada (San Martin-Rodriguez e col., 2005), comprovando a multiplicidade dos aspectos ligados à cooperação interprofissional e a necessidade de investigação para a identificação e compreensão de como cada determinante se relaciona com o outro nesse complexo fenômeno.

\section{Efeitos}

Uma revisão sistemática (Zwarenstein e col., 2009) foi realizada para avaliar o impacto de intervenções desenhadas para alterar a cooperação interprofissional. Foram incluídos ensaios controlados que apresentaram resultados relativos a desfechos ligados ao paciente, ao estado de saúde ou ao processo de assistência, obtidos por medidas objetivas ou autorreferidas, usando instrumento validado. Cinco estudos atenderam aos critérios de inclusão. Concluiu-se que as intervenções podem melhorar o processo e o resultado da assistência. Entretanto, considerando o número pequeno de estudos e sua heterogeneidade, não foi possível extrair inferências generalizáveis sobre elementos chaves da colaboração interprofissional e sua efetividade. Estudos mais rigorosos são necessários, incluindo métodos qualitativos que forneçam elementos de como as intervenções afetam a colaboração e como sua melhoria contribui para as mudanças nos desfechos.

Outra revisão (Martin e col., 2010) buscou investigar a base de evidência para a colaboração interprofissional envolvendo médicos, enfermeiras e novos modelos de cuidado. Foram incluídos 14 estudos. Os principais componentes das intervenções foram planos de tratamento, coordenação do cuidado, monitoramento do estado de saúde, treinamento no manejo do cuidado pelo paciente e promoção de serviços comunitários. As medidas eram indicadores reportados pelo paciente ou ligados à mortalidade, desfecho social, funcional e clínico e utilização de serviços médicos. Embora estudos mais rigorosos e em diferentes áreas sejam necessários, a base de evidência relacionada à colaboração interprofissio- nal mostrou resultados promissores em relação aos desfechos ligados ao paciente.

Os efeitos da prática cooperada podem alcançar desde uma melhor resolubilidade das ações (Oandasan e col., 2004; Gaboury e col., 2009), incluindo o aumento da diversidade de sua oferta (Gaboury e col., 2009, D`Amour e col., 2005) até a melhoria da comunicação entre trabalhadores, a otimização da participação da equipe na tomada de decisões e a potencialização do respeito entre os integrantes das equipes (Oandasan e col., 2004). Pode melhorar os resultados no cuidado a pacientes de grupos específicos (Strasser e col., 2008; Sinclair e col., 2009); usuários de serviços de práticas complementares (Gaboury e col., 2009); e de equipes de saúde da família (Goldman e col., 2010; Way e col., 2001). Outros estudos demonstram que equipes que cooperam entre si estarão mais aptas e capazes para coordenar a atenção, identificar as reais necessidades da população/comunidade e desenvolver novas tecnologias de atenção, produzindo respostas às demandas de saúde (Oandasan e col., 2004).

\section{Modelos Teóricos}

D’Amour e colaboradores (2005), analisando os modelos teóricos, em uma acepção estrita, identificaram dois estudos derivados da teoria organizacional: um apoiado na sociologia organizacional e outro na teoria da troca social. No primeiro, os pesquisadores propuseram um modelo que levava em consideração aspectos ligados à tarefa, à composição do grupo e aos contextos cultural e organizacional. Eles estudaram equipes do sistema de saúde do Reino Unido e os achados mostraram, entre outros aspectos, que equipes que trabalham bem, juntas, são mais efetivas e inovadoras. No segundo estudo foram investigados centros de saúde comunitária em Quebec (Canadá). Os resultados mostraram que a colaboração interprofissional depende das características do conflito, incluindo as complexidades das regras e dos compromissos profissionais.

No estudo apoiado na sociologia organizacional (D’Amour e col., 2008) o processo de colaboração é conceituado em quatro dimensões: finalização (objetivos compartilhados), que se refere à existência de objetivos comuns e o reconhecimento de motivações divergentes e múltiplos compromissos 
e sua apropriação pela equipe; interiorização (senso de pertencimento), dada pela consciência dos profissionais de sua interdependência; formalização (estruturação do cuidado clínico), por meio de protocolos que regulam a ação; e governança, que diz respeito às lideranças em níveis central e local, às expertises e ao grau de conexão entre elas. Esse modelo foi aplicado para analisar a colaboração entre profissionais, atuando em diferentes tipos de organização, que faziam parte de uma rede. Os resultados mostraram que maior grau de colaboração estava associado à forte liderança e a serviços mais acessíveis e contínuos (D’Amour e col., 2005).

Segundo os pesquisadores, a teoria da troca social parte da suposição de que as estruturas sociais podem ser entendidas pela análise das transações interpessoais. A compreensão das interações seria a chave para o entendimento dos comportamentos sociais complexos entre os grupos (D`Amour e col.. 2005). Devido ao foco da abordagem se voltar para o processo de troca e negociação entre indivíduos, os resultados pouco informam sobre dimensões organizacionais e estruturais associadas à cooperação interprofissional.

\section{Desafios para Futuros Estudos}

Para McDonald e McCallin (2010) quatro questões-chave permanecem como desafios. Como a colaboração interprofissional é um conceito multidimensional, para muitos profissionais ela é um objeto ainda obscuro, em relação ao qual não se sabe exatamente o que está envolvido. 0 segundo aspecto diz respeito aos desafios relacionados à presença de diferentes prestadores de serviços, o que torna mais complexa a problemática da definição de padrões para assegurar segurança e qualidade na assistência. Conhecimento das atribuições e responsabilidades e boa capacidade de comunicação são aspectos cruciais para uma colaboração interprofissional efetiva. Por fim, quase sempre os papéis do paciente e da família não estão claros na interação com e entre os profissionais, o que pode representar dificuldades adicionais em um modelo de prática colaborativa.

Em outra revisão (Mitchell e col., 2010) algumas proposições foram elaboradas a respeito dos processos associados à cooperação interprofissional, a fim de informar futuros estudos e prover um quadro mais compreensível sobre as implicações da composição e da dinâmica da equipe para sua efetividade. O impacto da diversidade profissional no desempenho da equipe foi examinado, reconhecendo que a habilidade para compartilhar conhecimento por fronteiras profissionais é um traço essencial para o desempenho de atividades complexas em equipes multiprofissionais. Entretanto, essa diversidade também engendra substanciais barreiras para a troca de conhecimentos e a solução de problemas, incluindo a falta de modelos mentais análogos, incompreensão semântica, conflitos epistemológicos e ameaças à identidade profissional. Uma das questões críticas seria examinar como os determinantes de nível micro e de nível macro atuam sobre o desempenho das equipes e compreender as relações entre e através dos contextos do cuidado à saúde.

\section{Novas Abordagens}

Analisando a pluralidade de conceitos, especialistas destacam a abordagem sistêmica como ponto comum dos modelos teóricos, no qual a cooperação interprofissional decorre de certos insumos ou ingredientes (inputs) e participa de um processo que gera efeitos (outputs). De modo resumido, os efeitos seriam aspectos relacionados à efetividade da equipe e os insumos, características associadas a fatores individuais, profissionais, organizacionais e estruturais (D'Amour e col., 2005).

O predomínio das abordagens sistêmicas tem representado para os estudos organizacionais uma dificuldade para a compreensão de processos socialmente construídos e, em grande medida, processos de mudança, os quais são tomados como problema que se coloca para um conjunto pré-existente, que só pode ser resolvido mediante a integração de elementos novos a esse conjunto. Assim, a mudança pode implicar tanto uma alteração quantitativa na magnitude do sistema quanto uma mudança qualitativa ou diferenciação estrutural, por meio de mecanismos que integram ou unificam as partes diferenciadas (Misoczky, 2003).

As organizações dependem do empenho de seus integrantes. Em se tratando de organizações de saúde isso se aplica de modo radical, pois, utilizando as expressões do autor, "são organizações 
profissionais, nas quais o saber e as habilidades são formalizados através do processo de formação e as normas definidas pelas associações profissionais" (Dussault, 1992, p. 11).

A cooperação interprofissional, em sua prática, requer o abandono de uma postura competitiva entre os profissionais (San Martin-Rodrigues e col., 2005). Porém, ao mesmo tempo em que os profissionais trabalham juntos para prover um melhor cuidado ao usuário, possuem interesses próprios e desejam manter certo grau de autonomia e independência profissional (D`Amour e col., 2008).

Os trabalhadores profissionais podem ser responsáveis por muitas decisões e tendem a preservar, com todo o zelo, os limites de autonomia possível em cada ato de seu trabalho. São trabalhadores diferenciados, particularmente pelo fato de que o próprio exercício das suas competências exige que disponham de certo grau de independência. Nenhum superior hierárquico pode impor ao profissional uma conduta determinada. Observa-se que os gerentes têm pouco poder sobre o trabalho dos produtores de serviços (os profissionais de saúde), os quais, devido ao monopólio do conhecimento e às competências adquiridas, definem as características do processo de trabalho sem o considerar, na maioria das vezes, como um desdobramento da missão e dos objetivos da organização (Dussault, 1992).

Se ao olhar para dentro das organizações é reconhecido que elas dependem do empenho de seus integrantes, ao olhar para fora observa-se que as organizações não estão isoladas completamente umas das outras. Elas podem interagir e frequentemente compartilham características do ambiente ou sistema de ação social. Um exemplo é o controle, a que os profissionais admitem se submeter, exercido por entidades corporativas (conselhos federais e regionais das profissões), cujos representantes foram escolhidos por eles mesmos.

A abordagem sistêmica se apoia na premissa de que a natureza da realidade social é um conjunto de fenômenos materiais ou de ideias, crenças e hábitos externos ao comportamento dos indivíduos. Essa abordagem tem sido amplamente utilizada para determinar o substrato social (as condições, as situações, os contextos, os padrões de comportamento), por meio do qual os atores sociais vivenciam e interagem. Mas esse tipo de análise não nos diz como os atores compreendem esse substrato, como percebem o papel dos outros e como moldam seus próprios comportamentos nem permite entender como a ação interativa dos atores sociais dotados de vontade (ainda que limitados por fatores estruturais) provoca mudanças (Misoczky, 2003).

Nesse sentido, outras abordagens serão necessárias se o que se busca é o foco em processos e em relações, adotando a premissa de que os sujeitos não são meros fenômenos da estrutura, mas agentes atuantes dotados de uma prática interessada, e que tanto os esquemas de percepção como as organizações e a estrutura possuem gênese social (Misoczky, 2003).

Tendo em vista os apontamentos citados anteriormente, a cooperação interprofissional é considerada como um fenômeno não totalmente compreendido, necessitando de mais estudos para apreender seus determinantes e seu impacto nos serviços. Há também poucas experiências de operacionalização da cooperação (Gaboury e col., 2009) que enfrentam dificuldades em sua implantação.

O interesse na temática da cooperação interprofissional tem crescido nas duas últimas décadas, em parte pelo aumento da complexidade do cuidado em saúde e pela melhor compreensão dos determinantes do processo saúde-doença. Também pelo aumento dos custos com o cuidado, advindos da especialização médica e do avanço tecnológico, que reforçam a necessidade de redesenhar as práticas em saúde. A prática colaborativa tem sido identificada como uma alternativa para a maior eficiência e para um cuidado apropriado (Gaboury e col., 2009).

Em virtude de sua complexidade, ainda são encontradas dificuldades na adoção da cooperação interprofissional como estratégia de trabalho. Dentre elas encontram-se: o cuidado fragmentado de sistemas tradicionais de saúde, a cultura profissional especializada, o sistema de saúde fragmentado e por categoria profissional e a baixa procura por carreiras na área da saúde, entre outros (WHO, 2010).

Atualmente, a cooperação interprofissional tem sido pauta nas discussões das políticas públicas, principalmente nos países ocidentais, com vistas à melhoria do acesso aos serviços, em uma atenção mais contínua e abrangente. Essa tendência em ado- 
tar novas formas de organização dos serviços requer não apenas a implementação de novas estruturas organizacionais, mas também o desenvolvimento de práticas clínicas compartilhadas (D`Amour e col., 2008).

Hall (2005) aponta outros desafios associados às culturas das profissões, identificando que os profissionais de saúde ainda não compartilham uma mesma cultura profissional, podendo surgir diversos conflitos relacionados com disputas de poder, de diferentes conceituações de saúde-doença, de diferentes valores e de diferentes contextos de aprendizagem.

As reformas do sistema de saúde destacam três dimensões críticas para a gestão do trabalho em saúde: as que envolvem aspectos relacionados com a gestão de sistemas e serviços de saúde; as vinculadas com questões relativas à formação e à disponibilidade dos profissionais; e as concernentes a mecanismos de interação entre o processo de trabalho e a legitimação profissional (Pierantoni, 2001).

Neste artigo interessa reter a dimensão ligada aos sistemas e serviços de saúde, na qual se reconhece a importância da gestão do trabalho para evitar a desumanização e os riscos na assistência à saúde associados à falta de compromisso e motivação dos profissionais; introduzir dispositivos para mediar os conflitos de interesse que muitas vezes surgem nos ambientes de trabalho, tanto dentro da própria equipe profissional como com os usuários; e contribuir para elevar a qualidade do cuidado, colocando no centro as necessidades do paciente.

Considerando que o corporativismo profissional é também um problema do sistema de saúde brasileiro, reconhecido por especialistas de diversos centros de pesquisa em saúde pública do país (Victora e col., 2011), é fortalecido o argumento de que a cooperação interprofissional deve ser incluída como um tema de investigação e um tópico na agenda dos responsáveis pela condução das políticas de saúde.

\section{Considerações Finais}

Com o aumento da complexidade e dos custos dos serviços associados às necessidades e expectativas dos usuários e dos trabalhadores, maior é a possi- bilidade de confusão, duplicação e ineficiência. Os administradores dos serviços sofrem maior pressão e a consequência disso é o aumento da sobrecarga que incide sobre os profissionais e demais trabalhadores da saúde. Seu efeito mais visível é a queda na qualidade da assistência dirigida aos usuários, polo mais frágil do processo.

O cuidado à saúde dos usuários é uma atividade cada vez mais complexa, que requer a interação dos profissionais de saúde. Essa interação pode assumir diferentes formas, uma das quais é a cooperação. Apesar de poucos estudos com evidências empíricas sobre a efetividade da prática colaborativa, seus resultados têm sido utilizados por gestores para melhorar as estratégias do trabalho com as equipes.

Os processos de mudanças para adoção da cooperação interprofissional nos serviços envolve a compreensão da complexidade desse fenômeno. Não apenas as questões organizacionais, mas sim a integração de diversos elementos que a determinam, como as relações entre os profissionais, os processos de trabalho e as condições da estrutura social e econômica que as várias organizações compartilham.

Os profissionais de saúde desempenham papel importante para a efetivação da cooperação, mas as características históricas da formação das profissões de saúde, entre outros determinantes, levam os profissionais a não atuarem de forma colaborativa. A compreensão de suas percepções acerca desse fenômeno, seus comportamentos e visões de mundo, bem como sua interação no ambiente de trabalho, podem ser elementos importantes para a identificação de estratégias de mudanças. Com as políticas de incorporação de novos profissionais no campo da APS descortinam-se espaços que podem favorecer o desenvolvimento de pesquisas e reflexões sobre a cooperação interprofissional, seja ela ou não um componente explicitado no desenho da organização.

Com base nos conhecimentos apresentados e diante das reformas em curso, considera-se que o estudo da interação profissional pode contribuir para a compreensão das forças que orientam o conflito e a cooperação interprofissional, subsidiando a formulação de estratégias para melhorar a produção do cuidado ao paciente no âmbito do sistema de saúde no Brasil. 


\section{Referências}

BALDWIN, D. M. C. Some historical notes on interdisciplinary teams and interprofessional education and practice in health care in USA. Journal of interprofessional care, London, v. 21, p. 23-37, 2007. Suplemento.

BORGES, C. F.; BAPTISTA, T. W. F. A política de atenção básica do Ministério da Saúde: refletindo sobre a definição de prioridades. Trabalho, Educação e Saúde, Rio de Janeiro, v. 8, n. 1, p. 2753, mar./jun. 2010.

BRASIL. Ministério da Saúde. Secretaria de Atenção à Saúde. Coordenação de Saúde da Comunidade. Saúde da família: uma estratégia para reorientação do modelo assistencial. Brasília: Ministério da Saúde, 1997.

BRASIL. Secretaria de Atenção à Saúde. Departamento da Atenção Básica. Diretrizes do NASF - Núcleo de Apoio à Saúde da Família. Cadernos de Atenção Básica 27, versão preliminar. Brasília: Ministério da Saúde, 2009.

BUSS, P. M. Promoção da saúde e qualidade de vida. Ciência \& Saúde Coletiva, Rio de Janeiro, v. 5 , n. 1, p. 163-167, 2000.

CAMPOS, G. W. S. Reforma política e sanitária: a sustentabilidade do SUS em questão? Ciência \& Saúde Coletiva, Rio de Janeiro, v. 12, n. 2, p. 301306, 2007.

CAMPOS, G. W. S.; DOMITTI, A. C. Apoio matricial e equipe de referência: uma metodologia para a gestão do trabalho interdisciplinar em saúde. Cadernos de Saúde Pública, Rio de Janeiro, v. 23, n. 2, p. 399-407, 2007.

CUETO, M. The origins of primary health care and selective primary health care. American Journal of Public Health, Washington, v. 94, n. 11, p. 18641874, 2004.

D`AMOUR, D. et al. The conceptual basis for interprofessional collaboration: core concepts and theorical frameworks. Journal of Interprofessional Care, London, p.116-131, 2005. Supplement.
D`AMOUR, D. et al. A model and tipology of collaboration between professionals in healthcare organizations. BMC Health Services Research, London, v. 8, n. 188, 2008.

DUSSAULT, G. A gestão dos serviços públicos de saúde: características e exigências. Revista de Administração Pública, Rio de Janeiro, v. 26, n. 2, p. 8-19, 1992.

FEELEY, N.; GOTTLIEB, L. N.; DALTON, C. The collaborative partnership approach to care: a delicate balance. Toronto: Elsevier Mosby, 2005.

FRANCO, T. B.; MERHY, E. E. Programa Saúde da Família (PSF): contradições de um programa destinado à mudança do modelo tecnoassistencial. In: MERHY, E. E. et al. $O$ trabalho em saúde: olhando e experenciando o SUS no cotidiano. São Paulo: Hucitec, 2007. p. 55-124.

GABOURY, I. et al. Interprofessional collaboration within Canadian integrative healthcare clinics: key components. Social Science \& Medicine, London, n. 69, p. 707-715, 2009.

GIOVANELLA, L.; MENDONÇA, M. H. M. Atenção primária à saúde. In: GIOVANELLA, L et al. (Org.). Políticas e sistema de saúde no Brasil. Rio de Janeiro: Editora Fiocruz, 20o8. p. 575-626.

GOLDMAN, J. et al. Interprofessional collaboration in family health teams: An Ontario-based study. Canadian Family Physician, Mississauga, n. 56, p. 368-374, 2010.

GOODMAN, B. Ms B and legal competence: interprofessional collaboration and nurse autonomy. Nursing in Critical Care, Oxford, v. 9, n. 6, p. 271-276, 2004.

HALL, P. Interprofessional teamwork: professional culture as barriers. Journal of Interprofessional Care, London, p.188-196, 2005. Supplement.

KENNY, G. Children's nursing and interprofessional collaboration: challenges and opportunities. Journal of Clinical Nursing, Oxford, v. 11, n. 3, p. 306-313, 2002.

LEATHARD, A. (Ed.). Interprofessional collaboration: from policy to practice in health and social care. Hove: Routledge, 2003. 
MARTIN, J. S. et al. Interprofessional collaboration among nurses and physicians: making a difference in patient outcome. Swiss Medical Weekly, 140: w13062, 2010. Disponível em <http:// www.smw.ch/for-readers/archive/backlinks/?url=/ docs/pdfcontent/smw-12648.pdf> Acesso em: 13 maio 2011.

McDONALD, C.; McCALLIN, A. Interprofessional collaboration in palliative nursing: what is the patient-family role? International Journal of Palliative Nursing, London, v. 16, n. 6, p. 286-289, 2010.

MENDES, E. V. Uma agenda para a saúde. São Paulo: Hucitec; 1996.

MENDES, E. V. A Atenção primária à saúde no SUS. Fortaleza: Escola de Saúde Pública do Ceará, 2002.

MISOCZKY, M. C. A. Implicações do uso das formulações sobre campo de poder e ação de Bourdieu nos estudos organizacionais. Revista de Ciências da Administração, Florianópolis, p.19-3o, 2003.

MITCHELL, R. et al. Review: toward realizing the potential of diversity in composition of interprofessional health care teams: an examination of the cognitive and psychosocial dynamics of interprofessional collaboration. Medical Care Research and Review, Thousand Oaks, v. 67, n. 1, p. 3-26, 2010.

MOROSINI M. V. G. C.; CORBO, A. D. Modelos de atenção e a saúde da família. Rio de Janeiro: EPSJV/Fiocruz, 2007.

NARASIMHAN, V. et al. Responding to the global human resources crisis. Lancet, Oxford, n. 363, p. 1469-1472, 2004.

NORONHA, J. C.; SOARES, L. T. A política de saúde no Brasil nos anos 9o. Ciência \& Saúde Coletiva, Rio de Janeiro, v. 6, n. 2, p. 445-45o, 2001.

OANDASAN, I. et al. Interdisciplinary education for collaborative, patient-centred practice. Ottawa: Health Canada, 2004.

ORGANIZACIÓN MUNDIAL DE LA SALUD. La atención primária de salud: más necessaria que nunca. Informe sobre la salud en el mundo. Genebra: OMS, 2008.
PAIM, J. S.; ALMEIDA-FILHO, N. Saúde coletiva: uma "nova saúde pública" ou campo aberto a novos paradigmas? Revista de Saúde Pública, São Paulo, v. 32, n. 4, p. 299-316, 1998.

PIERANTONI, C. R. As reformas do Estado, da saúde e recursos humanos: limites e possibilidades. Ciência \& Saúde Coletiva, Rio de Janeiro, v. 6, n. 2, p. 341-36o, 2001.

REESE, D. J.; SONTAG, M. A. Successful interprofessional collaboration on the hospice team. Health and Social Work, Oxford, n. 26, n. 3 , p. 167-175, 2001.

RIVERO, D. A. T. Alma-Ata: 25 años después. Perspectivas de Salud, Washington, v. 8, n. 2, p. 2-7, 2003.

SAN MARTIN-RODRIGUEZ, L. et al. The determinants of sucessfull collaboration: a review of theorical and empirical studies. Journal of Interprofessional Care, London, p. 132-147, 2005. Supplement.

SEVCENKO, N. A corrida para o século XXI: no loop da montanha-russa. São Paulo: Companhia da Letras, 2001.

SHAW, A.; LUSIGNAN, S.; ROWLANDS, G. Do primary care professionals work as a team: a qualitativa study. Journal of Interprofessional Care, London, v. 19, n. 4, p. 396-405, 2005.

SILVA, I. Z. Q. J.; TRAD, L. A. B. O trabalho em equipe no PSF: investigando a articulação técnica e a interação entre os profissionais. Interface Comunicação, Saúde e Educação, Botucatu, v. 9, n. 16, p. 25-30, 2004/2005.

SINCLAIR, L. B.; LINGARD, L. A.; MOHABEER, R. N. What's so great about rehabilitation teams? An ethnographic study of interprofessional collaboration in a rehabilitation unit. Archives of Physical Medicine and Rehabilitation, Reston, v. 90, p. 1196-1201, 2009.

SMITH, P. M.; SEELEY, J. A review of the evidence for the maximization of clinical placement opportunities through interprofessional collaboration. Journal of Interprofessional Care, London, v. 24, n. 6, p. 69o-698, 2010. 
STARFIELD, B.; SHI, L. Policy relevant determinants of health: an international perspective. Health Policy, Amsterdam, v. 6o, p. 201-218, 2002.

STRASSER, D. C. et al. Team training and stroke rehabilitation outcomes: a cluster randomized trial. Archives of Physical Medicine and Rehabilitation, Reston, v. 89, p. 10-15, 2008.

VICTORA, C. G. et al. Condições de saúde e inovações nas políticas de saúde no Brasil: o caminho a percorrer. The Lancet Series: health in Brazil, Oxford, p. 90-102, Maio/2011.

WAY, D. et al. Primary health care provided by nurse practitioners and family pshysicians in shared practice. Canadian Medical Association Journal, Mississauga, v. 165, n. 9, p. 1210-1214, 2001.

WORLD HEALTH ORGANIZATION. Framework for action on interprofessional education and collaborative practice. Genebra: WHO/HRH/ HPN/10.3, 2010. Disponível em: http://www.who. int/hrh/nursing_midwifery/en/. Acesso em: 12 fev. 2011.
YAN, J.; GILBERT, J.; HOFFMAN, S. WHO announcement - study group on interprofessional education and collaborative practice. Journal of Interprofessional Care, London, v. 21, n. 6, p. 588 589, 2007.

ZWARENSTEIN, M.; GOLDMAN, J.; REEVES, S. Interprofessional collaboration: effects of practice-based interventions on professional practice and healthcare outcomes. Cochrane Database Systematic Reviews, Oxford, v. 8, n. 3 , CDoooo72, 2009.

ZWARENSTEIN, M.; REEVES, S. Knowledge translation and interprofessional collaboration: Where the rubber of evidence-based care hits the road of teamwork. Journal of Continuing Education in the Health Professions, New York, v. 26, n. 1, p. 46-54, 2006. 